\title{
HOMEOMORPHISM GROUPS AND HOMOGENEOUS SPACES
}

\author{
JAN van MILL
}

\begin{abstract}
We show that the 2-sphere $S^{2}$ can be decomposed into two sets $A$ and $B$ such that (1) both $A$ and $B$ are connected, locally connected, dense and Baire, (2) both $A$ and $B$ are (much more than) topologically homogeneous, (3) $A$ is not homeomorphic to $B$, and (4) the autohomeomorphism groups of $A$ and $B$ are (algebraically) isomorphic.
\end{abstract}

1. Introduction. All spaces under discussion are separable metric. If $X$ is a space then $H(X)$ denotes the group of homeomorphisms of $X$ onto itself. In the literature much work has been done concerning the following question:

if $H(X)$ and $H(Y)$ are algebraically isomorphic, when are $X$ and $Y$ topologically homeomorphic?

Wechsler $[\mathbf{1 0}]$ showed that if $X$ and $Y$ are both nondiscrete and strongly $n$-homogeneous for all $n,{ }^{1}$ and if the algebraic isomorphism $\beta: H(X) \rightarrow H(Y)$ is also a topological homeomorphism when both $H(X)$ and $H(Y)$ are given the point-open topology, then $X$ and $Y$ are homeomorphic. The following result was subsequently obtained by Whittaker [11]: if $X$ and $Y$ are compact manifolds (with or without boundary) and if $\beta: H(X) \rightarrow H(Y)$ is an isomorphism, then there is a homeomorphism $f: X \rightarrow Y$ such that for all $h \in H(X)$ we have $\beta(h)=f \circ h \circ f^{-1}$. Among others, this shows that $X$ and $Y$ are homeomorphic. Rubin [9] has recently shown that in Whittaker's result the hypothesis "compact manifold" can be replaced by "locally compact and strongly locally homogeneous" (in fact Rubin shows much more). For related results see also $[\mathbf{3}$ and $\mathbf{4}, \mathbf{5}, \mathbf{6}]$.

These results suggest the following vague conjecture: if $X$ and $Y$ are "sufficiently homogeneous" then $X$ is homeomorphic to $Y$ if and only if $H(X)$ is isomorphic to $H(Y)$. Rubin's result implies that if $X$ is locally compact and strongly locally homogeneous then $X$ is sufficiently homogeneous. The aim of this note is to construct spaces with very strong homogeneity properties which are not "sufficiently homogeneous".

We will show that there is a 1-dimensional, connected, locally connected and dense subspace $A \subseteq S^{2}$ with, among others, the following properties:

(1) $A$ is strongly locally homogeneous,

(2) if $K \subseteq A$ is compact, then $A$ and $A \backslash K$ are homeomorphic, and

(3) if $K, L \subseteq A$ are compact, and if $f: K \rightarrow L$ is a homeomorphism, then $f$ can be extended to a homeomorphism $\bar{f}: A \rightarrow A$.

(4) $A$ is Baire.

Observe that (3) implies that $A$ is strongly $n$-homogeneous for all $n \in \mathbf{N}$. We

Received by the editors July 8, 1983 and, in revised form, December 21, 1983.

1980 Mathematics Subject Classification. Primary 54C35, 54H10, 54F20.

${ }^{1}$ For definitions see $\S 2$. 
also show that $B=S^{2} \backslash A$ has the same homogeneity properties, that $B$ is not homeomorphic to $A$, and that $H(B)$ and $H(A)$ are (algebraically) isomorphic. Our result shows that in Wechler's Theorem it is essential that the algebraic isomorphism is also a topological homeomorphism when the homeomorphism groups involved are given a suitable topology. In addition, we can also conclude that in Rubin's result the hypothesis "locally compact" cannot be replaced by "Baire". After having been informed about our result, Rubin asked whether "locally compact" can be replaced by "topologically complete". As far as I know, this is still open.

2. Preliminaries. A space $X$ is called strongly locally homogeneous if it has an open base $\mathcal{U}$ such that for each $U \in \mathcal{U}$ and points $x, y \in U$, there exists a homeomorphism $h \in H(X)$ with $h(x)=y$ and $h \mid X \backslash U$ equal to the identity. The most obvious examples of strongly locally homogeneous spaces are locally euclidean spaces. Clearly, every connected strongly locally homogeneous space is homogeneous.

Let $n \in \mathbf{N}$. A space is called strongly $n$-homogeneous if for all distinct $x_{1}, \ldots, x_{n}$ $\in X$ and all distinct $y_{1}, \ldots, y_{n} \in X$ there is a homeomorphism $h \in H(X)$ with $h\left(x_{i}\right)=y_{i}$ for all $i \leq n$.

A space $X$ is called a Bernstein set if each compact subspace of $X$ is at most countable. $Q$ denotes the space of rational numbers.

A cardinal is an initial ordinal, and an ordinal is the set of smaller ordinals. $c$ denotes $2^{\aleph_{0}}$.

3. Construction of $A$ and $B$. As usual, the 2-sphere $S^{2}$ is the space

$$
\left\{\left(x_{1}, x_{2}, x_{3}\right) \in \mathbf{R}^{3}: x_{1}^{2}+x_{2}^{2}+x_{3}^{2}=1\right\} \text {. }
$$

If $x \in S^{2}$ and $\varepsilon>0$, let

$$
B(x, \varepsilon)=\left\{y \in S^{2}: d(x, y) \leq \varepsilon\right\}
$$

(here $d$ refers to the euclidean metric on $\mathbf{R}^{3}$ ). Let $D$ be an arbitrary countable dense subset of $S^{2}$. Define

$$
B=\{B(x, q): x \in D, q \in Q \text { and } q>0\} .
$$

Observe that $B$ is countable.

For any $B \in B$ and for all finite $\mathcal{E}, \mathcal{F} \subseteq B$ having the same cardinality, say $\mathcal{E}=\left\{E_{1}, \ldots, E_{n}\right\}$ and $\mathcal{F}=\left\{F_{1}, \ldots, F_{n}\right\}$, such that $\cup \mathcal{E} \cup \cup \mathcal{F} \subseteq$ int $B$, if possible choose a homeomorphism $h: S^{2} \rightarrow S^{2}$ such that

(1) $h\left(E_{i}\right)=F_{i}$ for all $i \leq n$, and

(2) $h \mid\left(S^{2} \backslash B\right)=$ identity.

(Observe that this is always possible if $n=1$.)

Let $G \subseteq H\left(S^{2}\right)$ be the set of homeomorphisms obtained in this way. Observe that $G$ is countable. Let $s: S^{2} \rightarrow S^{2}$ be the antipodal mapping and let $\Phi \subseteq H\left(S^{2}\right)$ be the subgroup generated by $G \cup\{s\}$. A set $Y \subseteq S^{2}$ is called $\Phi$-stable if $h(Y)=Y$ for all $h \in \Phi$. If $x \in S^{2}$ then $V(x)=\{h(x): h \in \Phi\}$.

3.1. THEOREM. There is a set $A \subseteq S^{2}$ such that both $A$ and $B=S^{2} \backslash A$ are $\Phi$-stable and Bernstein.

Proof. Let $\left\{K_{\alpha}: \alpha<\mathfrak{c}\right\}$ enumerate all Cantor sets in $S^{2}$. By transfinite induction, for each $\alpha<\mathfrak{c}$ we will construct points $x_{\alpha}, y_{\alpha} \in K_{\alpha}$ such that

$$
\bigcup_{\beta \leq \alpha} V\left(x_{\beta}\right) \cap \bigcup_{\beta \leq \alpha} V\left(y_{\beta}\right)=\emptyset .
$$


Suppose that $x_{\beta}$ and $y_{\beta}$ are defined for all $\beta<\alpha$. Observe that $\left|\bigcup_{\beta<\alpha} V\left(x_{\beta}\right)\right| \leq$ $|\alpha| \cdot \aleph_{0}<\mathfrak{c}$ and similarly that $\left|\bigcup_{\beta<\alpha} V\left(y_{\beta}\right)\right|<\mathfrak{c}$. Since $\left|K_{\alpha}\right|=\mathfrak{c}$ and since $|\Phi| \leq \aleph_{0}$, it is easy to pick $x_{\alpha}$ and $y_{\alpha}$ satisfying $(*)$. For details see van Mill [7, §3].

Put $A=\bigcup_{\alpha<c} V\left(x_{\alpha}\right)$ and $B=S^{2} \backslash A$. Since $A$ is $\Phi$-stable and since $\Phi$ is a subgroup of $H\left(S^{2}\right)$, it easily follows that $B$ is $\Phi$-stable. Finally, both $A$ and $B$ are Bernstein sets since if e.g. $K \subseteq A$ is uncountable and compact, then $K$ contains a Cantor set which by construction intersects $B$. This is impossible.

We claim that $A$ and $B$ are the subsets of $S^{2}$ with the properties mentioned in the introduction. In the remaining part of this note we will prove this. First observe that both $A$ and $B$ are dense in $S^{2}$ (use that both $A$ and $B$ are Bernstein, or see the proof of Theorem 3.1). We will use this often without explicit reference in the remaining part of this note.

3.2. LEMMA. If $U \subset S^{2}$ is nonempty, open and connected, then both $U \cap A$ and $U \cap B$ are connected.

Proof. Suppose that e.g. $U \cap A$ is not connected. There are disjoint, nonempty open sets $V_{0}, V_{1} \subseteq U$ such that $A \cap U \subseteq V_{0} \cup V_{1}$. Then $K=U \backslash\left(V_{0} \cup V_{1}\right)$ separates $U$ and consequently has to be uncountable. Since $K$ is $\sigma$-compact, it therefore has to contain a Cantor set. But this Cantor set intersects $A$ since $B$ is Bernstein, a contradiction.

\subsection{COROLlaRY. Both $A$ and $B$ are connected and locally connected.}

\subsection{LEMMA. Both $A$ and $B$ are Baire.}

Proof. This is clear since both $A$ and $B$ intersect every Cantor set in $S^{2}$, and hence every dense $G_{\delta}$.

4. $H(A)$ and $H(B)$ are isomorphic. In this section we will show that $H(A)$ is isomorphic to $H(B)$ and also that $A$ is not homeomorphic to $B$. We use a technique due to Curtis and van Mill [1] , which also turned out to be useful in van Mill and Wattel [8].

4.1. LEMMA. If $h \in H(A)$, then $h$ can be extended to a homeomorphism $\bar{h}: S^{2} \rightarrow S^{2}$.

Proof. As in Curtis and van Mill [1] , put

$$
\{\bar{h}(x)\}=\bigcap_{n=1}^{\infty} \overline{h\left(U\left(x, 2^{-n}\right) \cap A\right)},
$$

where $U\left(x, 2^{-n}\right)=\left\{y \in S^{2}: d(x, y)<2^{-n}\right\}$. Notice that if $n \in \mathbf{N}$, then $U\left(x, 2^{-n}\right) \cap$ $A$ is connected, Lemma 3.2 , whence $Z=\bigcap_{n=1}^{\infty} \overline{h\left(U\left(x, 2^{-n}\right) \cap A\right)}$ is a decreasing intersection of subcontinua of $S^{2}$, and therefore must be a subcontinuum itself. Since $Z$ is obviously contained in $B$, and since compact subsets of $B$ are at most countable, $Z$ contains precisely one point. We conclude that $\bar{h}$ is well defined. It is easy to see that $\bar{h}$ is continuous.

Since by the same technique we can also extend $h^{-1}$, we conclude that $\bar{h}$ is a homeomorphism (the reader can also easily check directly that $\bar{h}$ is one-to-one).

In the same way one can prove the following lemma. 
4.2. LEMMA. If $h \in H(B)$, then $h$ can be extended to a homeomorphism $\bar{h}: S^{2} \rightarrow S^{2}$.

If $h \in H(A)$, then the extension $\bar{h} \in H\left(S^{2}\right)$ of $h$ is unique, since $A$ is dense in $S^{2}$. We can therefore define a function $\beta: H(A) \rightarrow H(B)$ by $\beta(h)=\bar{h} \mid B$.

\subsection{LEMMA. $\beta$ is an isomorphism.}

Proof. If $f, g \in H(A)$ then $\bar{f} \circ \bar{g}$ is an extension of $f \circ g$, and therefore has to be equal to $\overline{f \circ g}$. Similarly, if $f \in H(A)$, then $\bar{f}^{-1}$ is an extension of $f^{-1}$, whence it follows that $\bar{f}^{-1}=\overline{f^{-1}}$. From this it follows that $\beta$ is a homomorphism. Define $\gamma: H(B) \rightarrow H(A)$ by $\gamma(h)=\bar{h} \mid A$, where $\bar{h}$ is the extension of $h$ given by Lemma 4.2. By the same argumentation as above, $\gamma$ is a homomorphism. It is easy to verify that $\beta \circ \gamma=$ identity and that $\gamma \circ \beta=$ identity. We conclude that $\beta$ is an isomorphism.

Observe that the only thing we used for the proof of Lemma 4.3 is that both $A$ and $B$ are Bernstein.

We will now show that $A$ is not homeomorphic to $B$.

\subsection{LEMMA. $A$ and $B$ are not homeomorphic.}

PROOF. To the contrary, assume that $h: A \rightarrow B$ is a homeomorphsm. By using the same technique as in the proof of Lemma 4.1, we find that $h$ can be extended to a homeomorphism $\bar{h}: S^{2} \rightarrow S^{2}$. Observe that $\bar{h}(A)=B$ and $\bar{h}(B)=A$. Therefore, $\bar{h}$ has no fixed points. By the Brouwer-Poincaré Theorem, see Dugundji [2, p. 343], $\bar{h}$ must therefore send some point $x \in S^{2}$ onto its antipode $s(x)$. Suppose that without loss of generality $x \in A$. Since $\bar{h}(x) \in B$ and $\bar{h}(x)=s(x) \in A$ (recall that $s \in \Phi$ and that $A$ is $\Phi$-stable, Theorem 3.1), this is a contradiction.

5. Homogeneity properties of $A$ and $B$. In this section we will show that both $A$ and $B$ have the homogeneity properties which were announced in the introduction. We use a technique in van Mill [7, §4].

5.1. Lemma. Let $E \in B$ and choose $p, q \in \operatorname{int} E$ such that $p \in A$. There is a homeomorphism $h \in H\left(S^{2}\right)$ such that $h(A)=A \cup\{q\}, h(p)=q$ and $h \mid S^{2} \backslash E=$ identity.

Proof. For each $n \in \mathbf{N}$ we will construct an element $E_{n} \in B$ and an element $h_{n} \in \Phi$ such that

(1) $\operatorname{diam} E_{n}<2^{-n}$ and $E_{n} \subset$ int $E_{n-1} \subset E$,

(2) $q \in \operatorname{int} E_{n}$,

(3) $p \in h_{1}^{-1} \circ \cdots \circ h_{n}^{-1}\left(\right.$ int $\left.E_{n}\right)$ and $\operatorname{diam} h_{1}^{-1} \circ \cdots \circ h_{n}^{-1}\left(\operatorname{int} E_{n}\right)<2^{-n}$,

(4) $h_{n} \mid S^{2} \backslash E_{n-1}=$ identity.

Choose $E_{1} \in B$ such that $q \in \operatorname{int} E_{1} \subseteq E_{1} \subseteq$ int $E$ while moreover diam $E_{1}<\frac{1}{2}$. There is an element $V_{1} \in B$ such that $p \in \operatorname{int} V_{1} \subseteq V_{1} \subseteq$ int $E$ such that also $\operatorname{diam} V_{1}<\frac{1}{2}$. By construction, there is an element $h_{1} \in \Phi$ such that $h_{1}\left(V_{1}\right)=E_{1}$ while $h_{1}$ restricts to the identity on $S^{2} \backslash E$. This defines $E_{1}$ and $h_{1}$. Suppose now that we have constructed $h_{i}$ and $E_{i}$ for all $i \leq n$." Choose $F_{0} \in B$ such that $h_{n} \circ \cdots \circ h_{1}(p) \in \operatorname{int} F_{0} \subseteq F_{0} \subseteq \operatorname{int} E_{n}$, while moreover diam $h_{1}^{-1} \circ \cdots \circ h_{n}^{-1}\left(\operatorname{int} F_{0}\right)<$ $2^{-(n+1)}$. In addition, let $F_{1} \in B$ be such that $q \in \operatorname{int} F_{1} \subseteq F_{1} \subseteq$ int $E_{n}$ and $\operatorname{diam} F_{1}<2^{-(n+1)}$. By construction, there is an element $g \in \Phi$ with $g\left(F_{0}\right)=F_{1}$ 
and $g \mid\left(S^{2} \backslash E_{n}\right)=$ identity. Define $h_{n+1}=g$ and $E_{n+1}=F_{1}$. This completes the induction.

Observe that if $d(x, p)>2^{-n}$ then $h_{n} \circ \cdots \circ h_{1}(x) \notin E_{n}$ and consequently, by (1) and (4),

$$
h_{k} \circ \cdots \circ h_{1}(x)=h_{n} \circ \cdots \circ h_{1}(x) \text { for all } k \geq n .
$$

This implies that if we define $h: S^{2} \rightarrow S^{2}$ by $h=\lim _{n \rightarrow \infty} h_{n} \circ \cdots \circ h_{1}$, then $h$ is well defined. Observe that $h(p)=q$ and that $h$ restricts to the identity on $S^{2} \backslash E$. The easy check that $h$ is a homeomorphism is left to the reader. Choose $x \in A$ arbitrarily. If $x=p$ then $h(x)=q$. If $x \neq p$, then $h(x) \in A$, since $A$ is $\Phi$-stable. We conclude that $h(A) \subseteq A \cup\{q\}$. Choose $y \in A$ arbitrarily. If $y=q$ then $h(p)=y$. If $y \neq q$, then find an index $n \in \mathbf{N}$ such that $y \notin E_{n}$. Let $x=h_{1}^{-1} \circ \cdots \circ h_{n}^{-1}(y)$. It is clear that $x \in A$ and that $h(x)=y$. We conclude that $A \cup\{q\} \subset h(A)$.

This proves that $h$ is as required.

\subsection{COROLlary. A is strongly locally homogeneous.}

Take $p \in A$ and $q \in B$. By Lemma 5.1 there is a homeomorphism $h: S^{2} \rightarrow S^{2}$ such that $h(p)=q$ and $h(A)=A \cup\{q\}$. Consequently, $h(A \backslash\{p\})=A$. We conclude that $A$ is homeomorphic to $A \backslash\{p\}$. Since every compact subset of $A$ is countable, and hence can be covered by a finite pairwise disjoint subcollection of $B$ of arbitrarily small mesh, the reader can easily verify that the following generalization of Lemma 5.1 holds: let $E \in B$ and choose compacta $K, L \subseteq$ int $E$ such that $K \subseteq A$; if $f: K \rightarrow L$ is a homeomorphism, then there is a homeomorphism $h \in H\left(S^{2}\right)$ such that $h(A)=A \cup L$ while moreover $h(x)=f(x)$ for every $x \in K$ (the proof of this fact needs homeomorphisms of a different type than the ones used in the proof of Lemma 5.1, but we made sure that the required homeomorphisms are available, see the definition of $\Phi$ in $\S 3$ ). Consequently, each compact subspace of $A$ can be pushed out of $A$ and homeomorphisms between compact subsets of $A$ extend. This more or less completes the proof of the following

5.3. THEOREM. If $K \subseteq A$ is compact, then $A$ is homeomorphic to $A \backslash K$. If $K, L \subseteq A$ are compact, and if $f: K \rightarrow L$ is a homeomorphism, then $f$ can be extended to a homeomorphism $\bar{f}: A \rightarrow A$.

It is clear that the required homogeneity properties of $B$ follow by precisely the same argumentation.

6. Remarks. M. Rubin has observed that every isomorphism of $H(A)$ is inner. E. K. van Douwen has constructed an infinite-dimensional version $C$ of $A$ such that $H(C)$ has an isomorphism that is not inner. This space is a subspace of the product $\left(S^{1}\right)^{\infty}$ and is homeomorphic to its complement.

\section{REFERENCES}

1. D. W. Curtis and J. van Mill, Zero-dimensional countable dense unions of $Z$-sets in the Hilbert cube, Fund. Math. 118 (1983), 103-108.

2. J. Dugundji, Topology, Allyn and Bacon, Boston, Mass., 1966.

3. N. J. Fine and G. E. Schweigert, On the group of homeomorphisms of an arc, Ann. of Math. 62 (1955), 237-253.

4. M. Gerstenhaber, Canonical constructions. I, Proc. Nat. Acad. Sci. U.S.A. 41 (1955), 233-236.

5. __ Canonical constructions. II, Proc. Nat. Acad. Sci. U.S.A. 42 (1956), 881-883. 
6. __ Canonical constructions. IV, Proc. Amer. Math. Soc. 8 (1957), 745-749.

7. J. van Mill, Strong local homogeneity does not imply countable dense homogeneity, Proc. Amer. Math. Soc. 84 (1982), 143-148.

8. J. van Mill and E. Wattel, Partitioning spaces in homeomorphic rigid parts, Colloq. Math. (to appear).

9. M. Rubin, On the reconstruction of topological spaces from their groups of homeomorphisms, Trans. Amer. Math. Soc. (to appear).

10. M. T. Wechsler, Homeomorphism groups of certain topological spaces, Ann. of Math. 62 (1955), 360-373.

11. J. V. Whittaker, On isomorphic groups and homeomorphic spaces, Ann. of Math. 78 (1963), 74-91.

Subfaculteit Wiskunde, Vrije Universiteit, De BoelelaAn 1081, Amsterdam, THE NETHERLANDS

Mathematisch InstituUt, Universiteit VAN Amsterdam, RoeterstraAt 15, AmsterdaM, THE Netherlands 